\title{
STRESS AND STRAIN TENSORS IN GRANULAR MEDIUM APPLICATION TO MASONRY STRUCTURES
}

\author{
Brahim CHETOUANE/ Ecole des Mines d'Alès, \\ CMGD (France)
}

\author{
Frédéric DUBOIS/ University of \\ Montpellier II LMGC (France)
}

\author{
Claude BOHATIER/ University of Montpellier II LMGC \\ (France)
}

\author{
Marc VINCHES/ Ecole des Mines \\ d'Alès, CMGD (France)
}

\begin{abstract}
The general framework of the present paper is the modeling of stone work, subject to dynamic loads. In this study, a masonry structure is considered as a collection of rigid blocks, linked together by Coulomb's type friction laws. We define on such a granular medium, a stress tensor and a macroscopic strain tensor, on an elementary cell. The definition of these tensors allows us to get, locally, an average information on this granular medium.
\end{abstract}

\section{INTRODUCTION}

Unlike continuous media, a discrete medium is characterized by the existence of contacts or interface between the discrete elements, constituting the system. The modeling using discrete elements, describes the contact forces and the displacements of each of these grains. The calculation algorithm is incremental. From the detection of contacts, are determined the displacements and contact forces at the end of each time step, for all the grains. At the following step, new positions are updated, new contact forces computed, and the process goes on. This general principle is the basis of the calculation using discrete elements. We decided to follow the works of M. JEAN and J.J. MOREAU [1] , embedded in the LMGC90(*) code.

First, an average stress tensor is defined on a cell, averaging the contact forces applied on each grain. Then, an average strain tensor is defined, using the cinematic description obtained from the discrete modeling of the grain system.

As an example, we present, as a test, the 2D results, obtained from LMGC90, on a 51 rectangular block wall, each block being $30 \mathrm{~cm}$ long, and $20 \mathrm{~cm}$ high. We then present the behavior of a segment of the Arles aqueduct, (248 blocks in the walls, and 98 blocks in the arch).

\section{NOMENCLATURE}

Granular materials, stress, strain.

\section{I- STRAIN TENSOR IN A CELL OF THE GRANULAR SAMPLE :}

The definition of a strain tensor remains a subtle notion in the case of a collection of rigid grains, each of the grain being subject to no strain. The aim of this tensor or indicator, is to describe the re-arrangement of the blocks versus time. We can get an information about the eventual sliding between grains, using the deviator component of this tensor. The volumetric strain, reflects the opening of joints and extension of the sample.

BAGI et al[3] chose a purely geometrical definition of this tensor, consisting in the definition of strain from the displacement of the center of gravity of the grains. Massao SATAKE [4] considered the same definition but adopted a Dirichlet's tessellation of the granular collection.

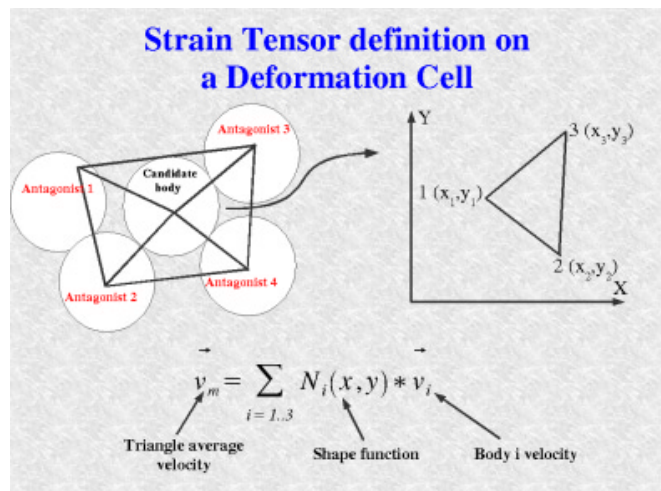

Fig. I.1 : Deformation cell definition 
The cell being defined as shown on fig. I.1, the average speed gradient is computed using the following formula :

$$
\left\langle\mathrm{v}_{\mathrm{i}, \mathrm{j}}\right\rangle=\frac{1}{\mathrm{~V}} \int_{\Omega}\left(\mathrm{v}_{\mathrm{i}, \mathrm{j}}\right) \mathrm{d} \Omega
$$

The calculation of the strain tensor uses the triangles of $\mathrm{Gi}$ summit, and opposed edge GlGk.

The speed of a point of the triangle is given by the following linear interpolation :

$$
\mathrm{v}_{\mathrm{i}}^{\text {triangle }}=\sum_{\alpha=1,3} \mathrm{~N}_{\alpha} \mathrm{V}_{\mathrm{i}}^{\alpha}
$$

with: $\mathrm{N}_{\alpha},(\alpha=1 . .3)$, interpolation functions at node $\alpha$. Thus,

$$
\mathrm{v}_{\mathrm{i}, \mathrm{j}}^{\text {triangle }}=\sum_{\alpha=1,3} \mathrm{~N}_{\alpha, \mathrm{j}} \mathrm{V}_{\mathrm{i}}^{\alpha}
$$

The tensor of strain speeds is the symmetrical part of the speed gradient : :

$$
\dot{\varepsilon}_{\text {triangle }}=\frac{1}{2}\left(\left\langle\mathrm{v}_{\mathrm{i}, \mathrm{j}}^{\text {traingle }}\right\rangle+\left\langle\mathrm{v}_{\mathrm{j}, \mathrm{i}}^{\text {triangle }}\right\rangle\right)
$$

Once calculated for each triangle, the strain speed, the total strain speed of the gauge can be written as follows:

$$
\dot{\varepsilon}=\frac{1}{\mathrm{~V}_{\text {jauge }}} \sum_{\text {triangles }} \stackrel{\bullet}{\varepsilon}_{\text {triangle }} \cdot \mathrm{V}_{\text {triangle }}
$$

\section{II- STRESS TENSOR FOR A GRANULAR MEDIUM :}

Most authors, interested in the definition of stress tensors in a granular medium, have developed their tools in a quasi-static case.[1] [4] [6] [9]

In the case of dynamics and in absence of rotations, the tensor of average constraint defined on the grain $\mathrm{S}$ of volume $\Omega$ is written as follows [1],[11] :

$$
\left\langle\sigma_{i j}\right\rangle=\frac{1}{\Omega} \sum_{\alpha}\left(x_{i}^{\alpha} f_{j}^{\alpha}-x_{i}^{\alpha} m_{i} \gamma_{i}\right)
$$

where $\mathrm{f}_{\mathrm{i}}^{\alpha}$ is the external force applied on point $\mathrm{x}_{\mathrm{i}}^{\alpha}$ and $\gamma_{\mathrm{i}}$ the acceleration of the grain $\mathrm{i}$ de masse $\mathrm{m}_{\mathrm{i}}$.

In this case where the external power equals the internal power, the internal momentum equals the momentum of the externals forces. Without any rotation, this tensor is symmetrical.

The external forces, are reduced to contact forces and dead weight. The tensor is defined taking as a reference whatever point in the space.

Lets consider, here under (fig. II.1), a grain $\mathrm{S}$ in contact with another grain $\mathrm{S}^{\prime}$ at point I

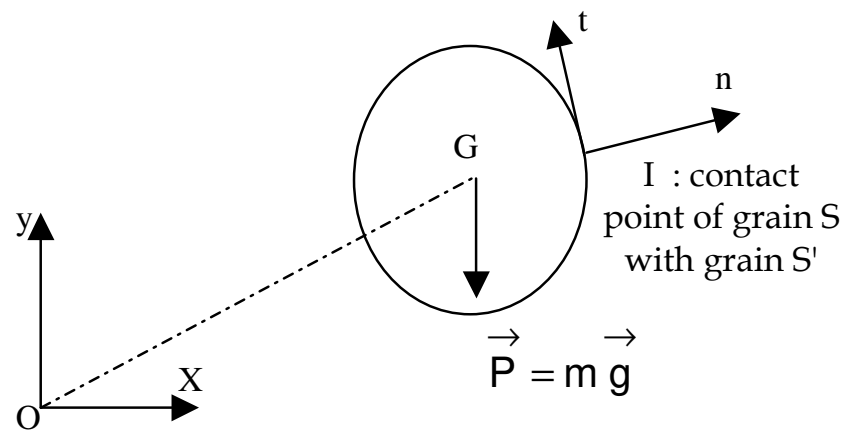

Fig. II.1 : Stress computing Principle

The stress tensor, computed with respect to point $\mathrm{O}$, can be written as follows :

$$
\langle\sigma\rangle=\frac{1}{\operatorname{vol}(\Omega)}[\overrightarrow{\mathrm{OI}} \otimes \overrightarrow{\mathrm{R}}+\overrightarrow{\mathrm{OG}} \otimes \overrightarrow{\mathrm{P}}-\mathrm{mOG} \otimes \vec{\gamma}](7)
$$

And, because of the fundamental principle of dynamics, $\rightarrow \rightarrow \rightarrow$

$\mathrm{P}+\mathrm{R}=\mathrm{m} \gamma$, the choice of the origin does not matter, and then, the expression of this average stress tensor can be reduced to :

$$
\langle\sigma\rangle=\frac{1}{\operatorname{vol}(\Omega)}[\overrightarrow{\mathrm{GI}} \otimes \overrightarrow{\mathrm{R}}]
$$

The dynamic term due to rotations destroys the symmetry of the stress tensor [4].

In the rest of the present paper, we will be in the case of small rotations, so that the symmetry of the stress tensor is ensured, and so that the stress tensor expression (8) can be used.

Equation (8) gives us the expression of a stress tensor on a grain. Using the additive property of this tensor, in the case of a discrete collection of grains, one can define the stress on the cell defined on fig. I.1. follows:

The stress tensor, on this cell, will then be written as

$$
\left\langle\sigma_{\text {jauge }}\right\rangle=\frac{1}{V_{\text {jauge } 1 . .\left(\mathrm{n}_{\mathrm{c}}+1\right)}\langle\sigma\rangle \cdot \mathrm{V}_{\text {grain }}}
$$

where $n_{c}$ is the number of grains in contact with the central grain $\mathrm{S}$.

For this stress tensor, one can determinate the associated Eigen values and vectors, giving respectively the principal stresses and associated principal stress directions.

\section{III- APPLICATION EXAMPLES :}

\section{III-1-Modeling of a wall under periodic solicitation :}

The preceding definitions of the strain and stress tensors were introduced in the LMGC90 code. The following results 
correspond to a wall, composed of 248 rectangular blocks, $30 \mathrm{~cm}$ long $x 20 \mathrm{~cm}$ high, laid on the ground. All block and the ground are considered rigid, and the ground has a sinusoidal uniform movement in the horizontal direction. The sinusoidal speed of the wave is applied with an amplitude $1 \mathrm{~m} . \mathrm{s}^{-1}$, a frequency of 2 $\mathrm{Hz}$, for a duration of 1 second.

As the ground starts moving, the triangulation described on fig.III.1 is subject to deformation. Then, maps showing different levels of the invariant of the strain were produced. We first plotted the instantaneous volume strain versus time. This invariant gives us information about the eventual dilation happening in the structure. More over, using the color distribution, we get an information about the localization of strains and joints openings (fig. III.2).

We then plotted the deviator part of the cumulated strain. This parameter shows us the relative importance of the sliding phenomena, happening in the structure (fig. III.3).

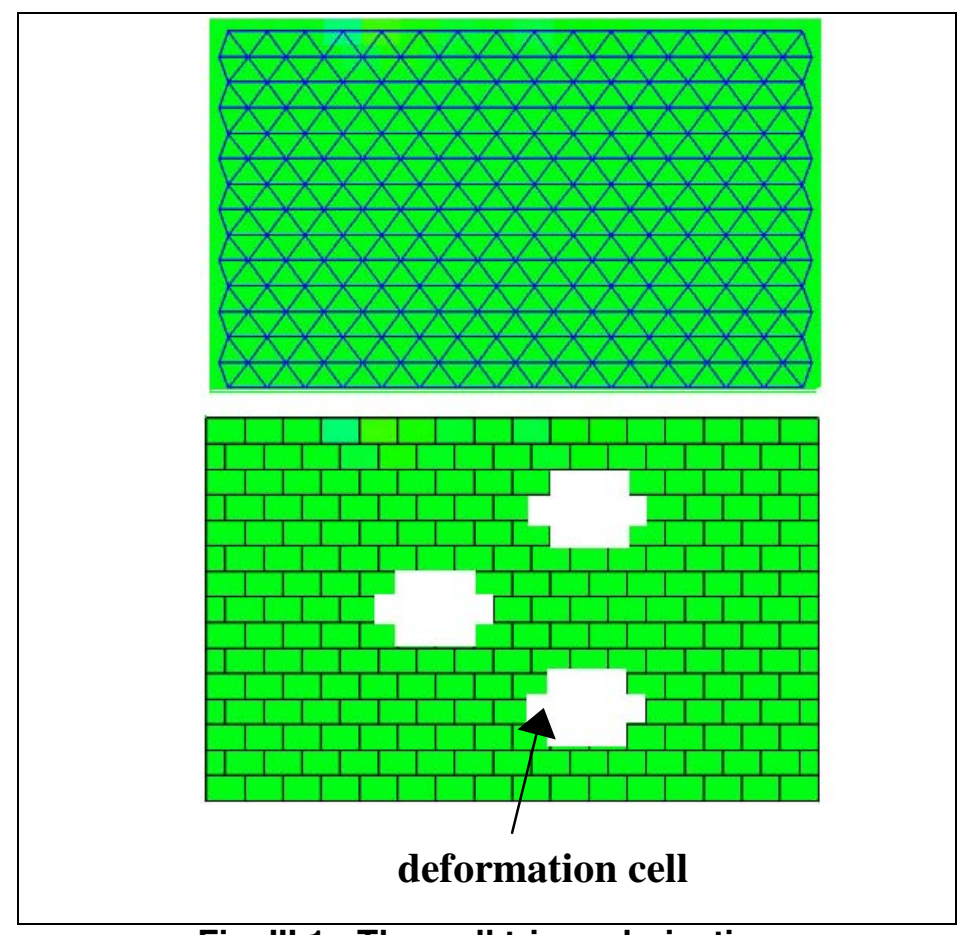

Fig. III.1 : The wall triangularisation and the cell definition

On those figures, one can observe that the mesh deformation occurs with both joint openings and the volume dilatation of the structure.

Figure III.2 shows that the localization of deformations corresponds to the important opening of joints.

Last, one can say that the instantaneous volume deformation, during this loading periodical process, is not an increasing function. This confirms the phenomenon of opening and closing of joints.

Similarly, stress contours have been produced. Fig. III.4 presents the evolution versus time, of the spherical invariant of the stress tensor, on the cell. Fig. III.5 shows the deviatory part of the stress tensor.

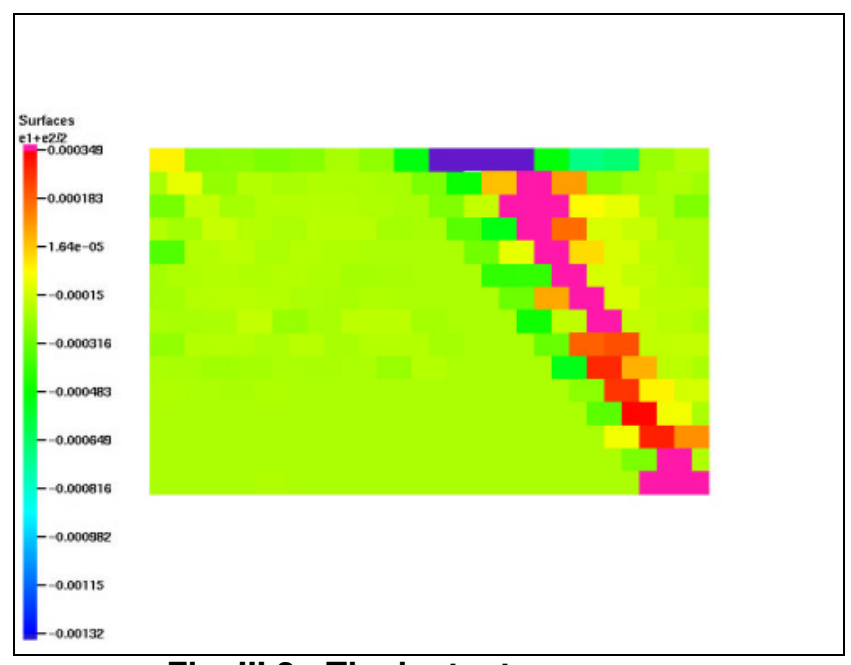

Fig. III.2 : The instantaneous volume strain at $t=0.1$ second

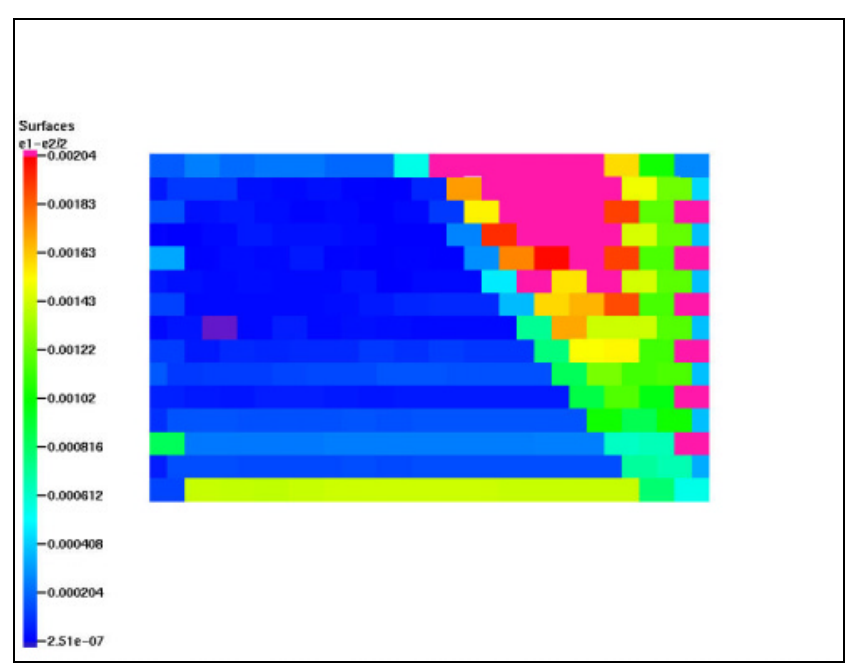

Fig. III.3 : The deviatoric component of the strain tensor at $t=0.1$ second

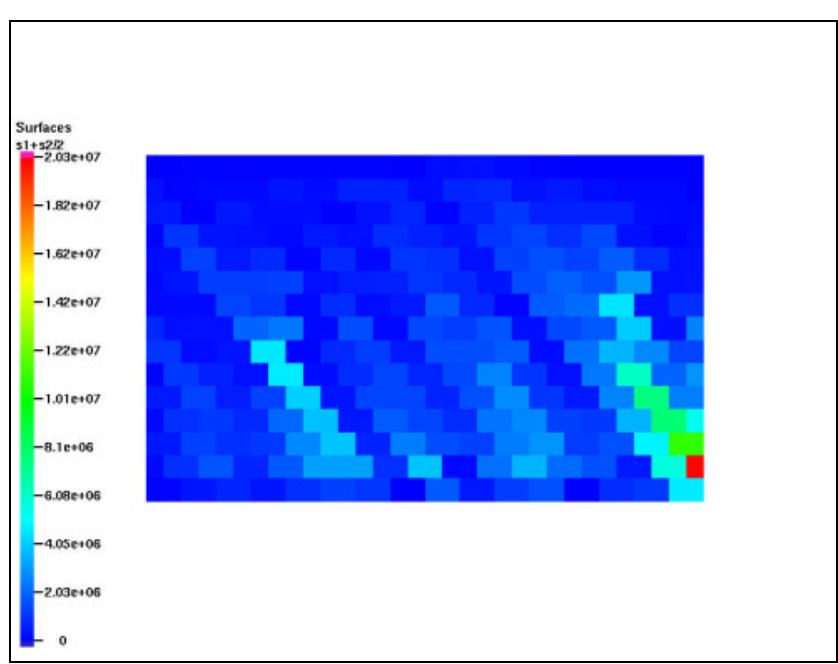

Fig. III.4 : The spherical component of the stress tensor at $t=0.1$ second 


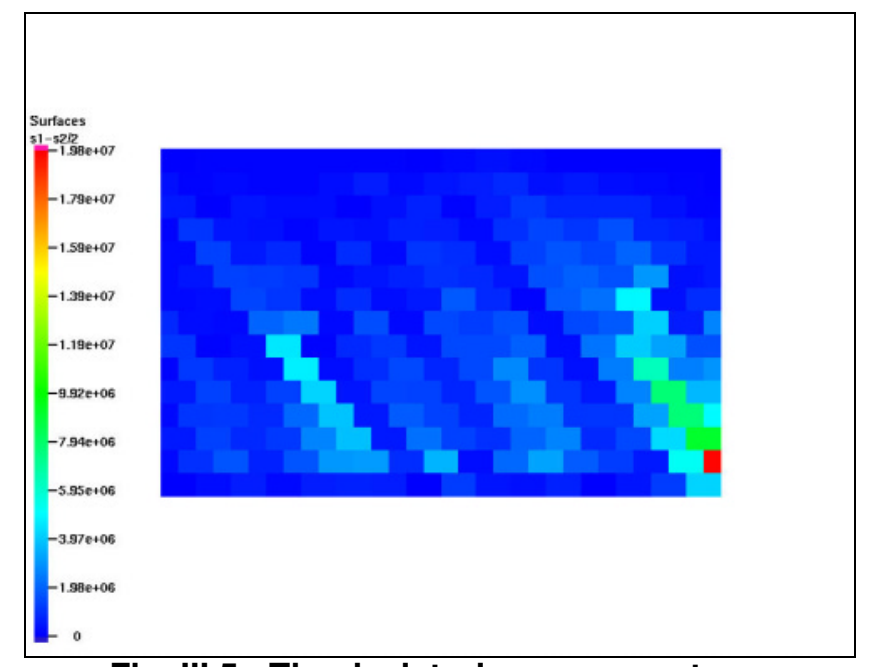

Fig. III.5 : The deviatoric component of the stress tensor at $t=0.1$ second

\section{III-2- Modeling of a stone arch submitted to a relative settlement :}

In this part we consider a stone arch composed of 98 rigid blocks. The calculation is usually done by Non Smooth Contact Dynamics Approach [1] programmed in LMGC90 software. The friction coefficient between different blocks is equal to 0.6. The friction coefficient at the interface between the ground and the structure is also equal to 0.6 . The density of the stone blocks is equal to $2500 \mathrm{~kg} \cdot \mathrm{m}^{-3}$ and the ground is supposed rigid and has a density of $1650 \mathrm{~kg} \cdot \mathrm{m}^{-3}$.

The stone arch is submitted to a relative settlement as shown in fig.III.6. The right pile is subjected to a vertical velocity equal to $-1 \mathrm{~cm} \cdot \mathrm{s}^{-1}$.

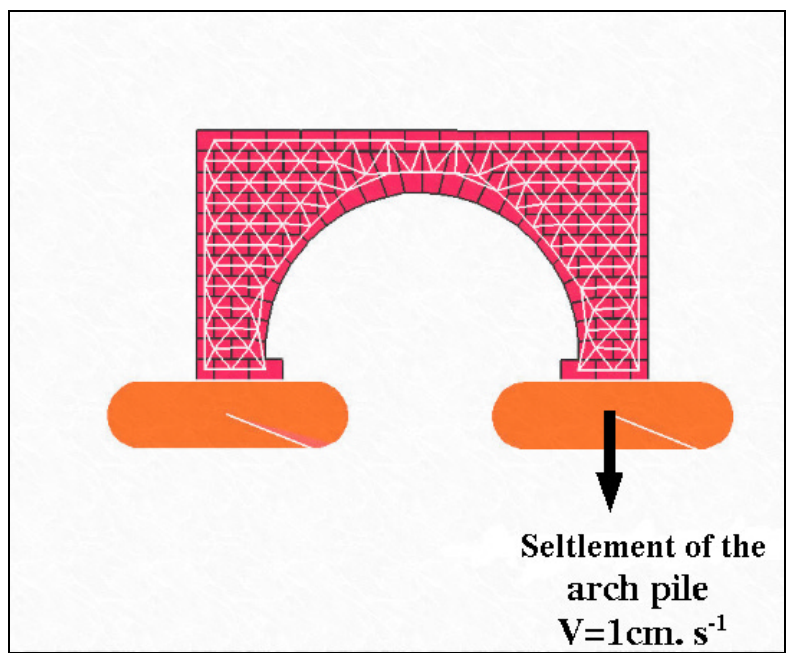

Fig. III.6 : Arch geometry and solicitation definition

The figures below show the different invariants of the two tensors at $\mathrm{t}=5$ seconds. We can notice that a great part of the left pile is under the highest level of a volume strain. The most solicited blocks are those who form the arch of the bridge. They are more compressed and the sliding ratio (Fig.III. 10) is higher for them than for the other blocks.

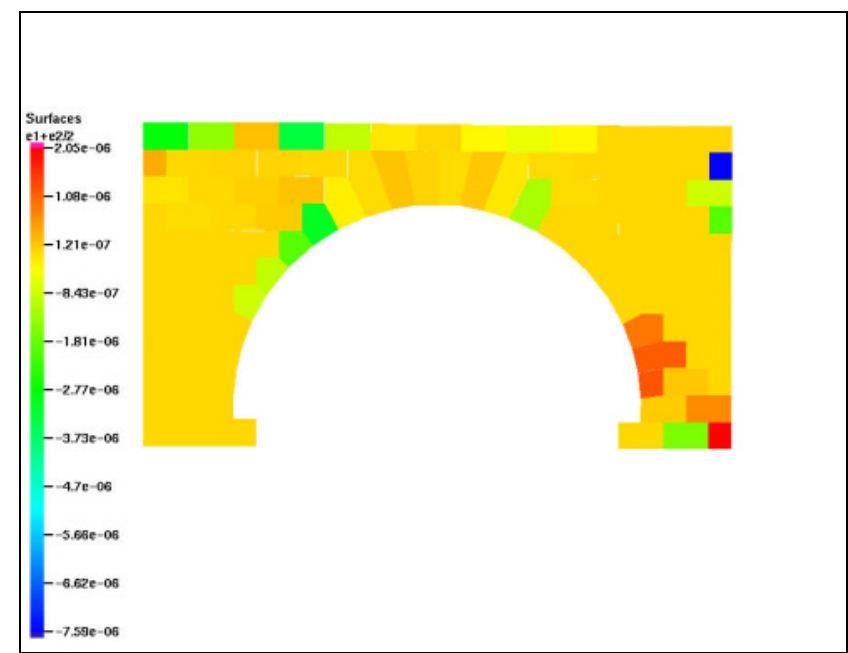

Fig. III.7 : The instantaneous volume strain at $t=5$ seconds

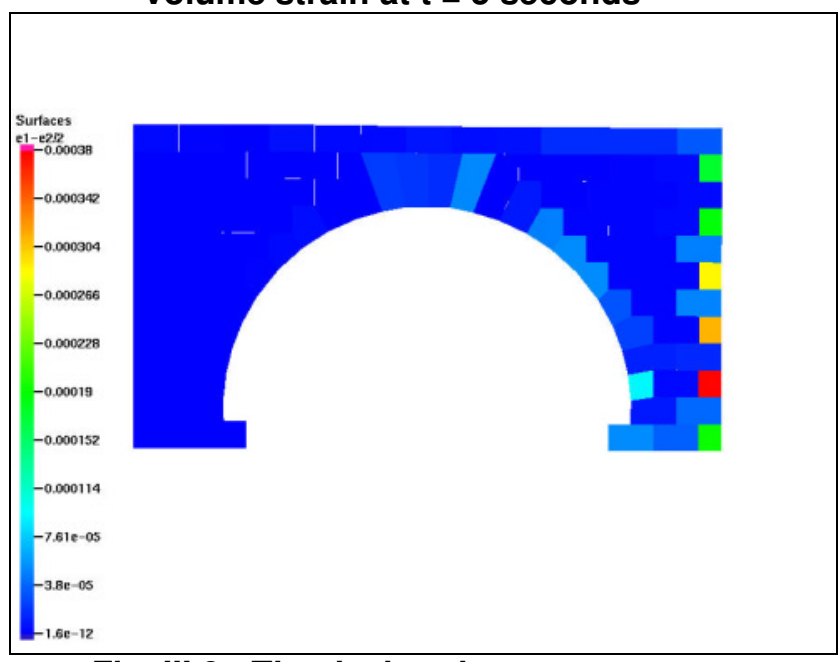

Fig. III.8: The deviatoric component of the strain tensor at $t=5$ seconds

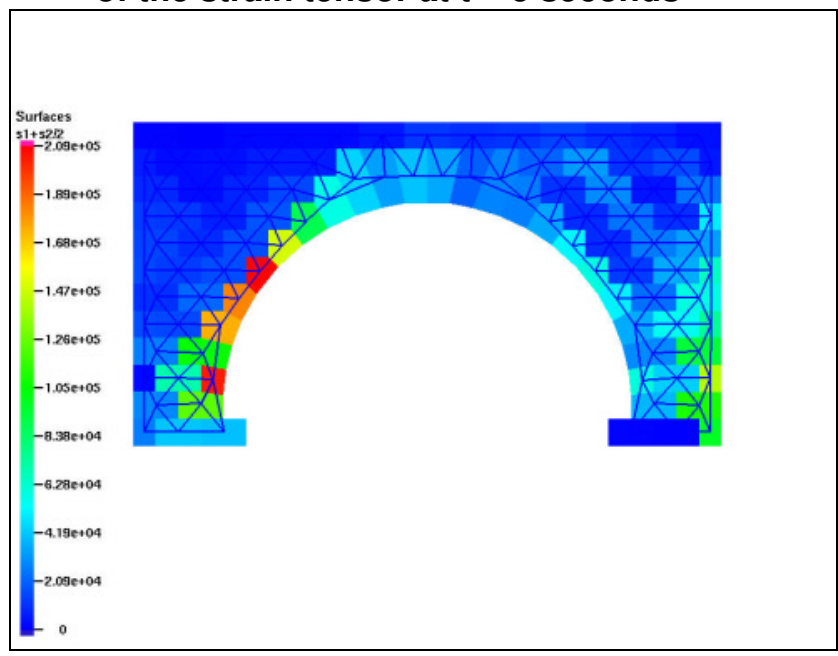

Fig. III.9 : The spherical component of the stress tensor at $t=5$ seconds 


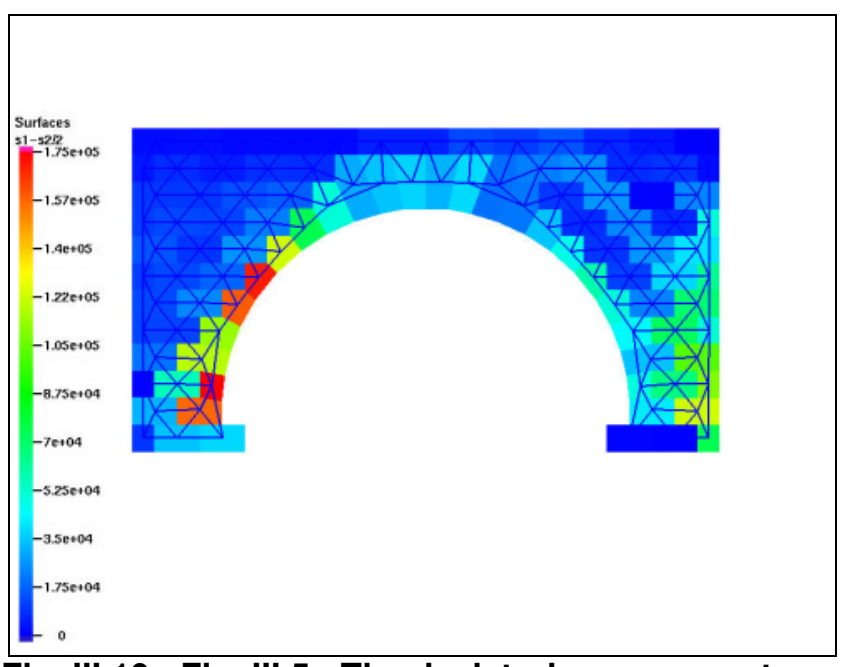

Fig. III.10 : Fig. III.5 : The deviatoric component of the stress tensor at $t=5$ seconds

\section{IV- CONCLUSION :}

The approach, presented in this paper, allows us to draw preliminary conclusions on the dynamic behavior of masonry structures. It is a first step of a phenomenological approach, to study in more details, the mechanisms involved in this type of structures.

The pros of such an approach are that one can get both information about the discrete and continuous nature, the local and global evolution of the structure. The main difficulty of this type of approach is that, for important rotations, the symmetry of the stress tensor cannot be obtained.

A representation of the load level on a grain, with respect to a given failure criterion, is sufficient, as a first step, to conclude on a possible failure. The more heavily loaded grains could be isolated from the rest of the structure and studied using the classical framework of continuous media mechanics. This engineering type approach is used for the analysis of engines mechanisms, in which solids can first be considered as rigid, and where deformations are then considered, in a basis associated with the rigid solid.

The following steps of the work to come will deal with the exploitation of the results of the developments described above.
We will try and define adequate approximations to obtain a generalization of the stress tensor, in order to be able to deal with the behavior of masonry discontinuous structures, under more general dynamic loading conditions. The modeling of the complete Arles aqueduct, at the Vallon des Arcs will be completed, in order to compare different failure hypothesis, formulated by archaeologists.

\section{REFERENCES :}

[1] JEAN, M. , CAMBOU, C. «Micromécanique des matériaux granulaires $\gg$ HERMES Science.

[2] JEAN, M. (1999) «The non-smooth contact dynamics method » Computer methods in applied mechanics and engineering. [Comput. methods appl. mech. eng.. ] 1999 , vol. 177 , no 3-4 , pp. 235 - 257 [ 23 pages. ]

[3] MOREAU, J. J. and JEAN, M. (1992) "Unilaterality and dry friction in the dynamics of rigid bodies collections" Proceedings of Contact Mechanics International Symposium, A. Curnier, ed., Lausanne. Presses Polytechniques et Universitaires Romandes, 31-48.

[4] MOREAU, J. J. « Numerical investigation of shear zones in granular materials », Proc. HLRZ-Workshop on friction, Arching, Contact Dynamics, World Scientific, Singapore, 1997, pp233-247, (Grassberger, P. et Wolf, D. eds).

[5] RENON, N. "Simulation numérique par éléments finis des grandes deformations des sols. Application à la stratification", Thèse de doctorat ENSMP, CEMEF, Sophia.

[6] BAGI, K. (1995-6), "Geomaterial Modeling of Granular Assemblies", Acta Technica Acad. Sci. Hung. 107(1-2), 1-16.

[7] BAGI, K. (1996), "Stress and Strain in Granular Assemblies," Mechanics of Materials, 22, 165-177.

[8] KRUYT, N.P. and L. ROTHENBURG (1996), "Micro mechanical Definition of Strain Tensor for Granular Materials," Journ. Applied Mech., Transactions of the ASCE, 118, 706-711

[9] ODA, M. and Iwashita (Editors) (1999), Mechanics of Granular Materials, An Introduction, A.A. Balkema, Rotterdam.

[10] SATAKE, M. (2001), "Stress and Strain in Granular Materials" in Powders \& Grains 2001 (ed. Y.Kishino), A.A.Balkema, Lisse, 149-152.

[11] FORTIN, J. "Simulation numérique de la dynamique des systèmes multicorps appliquée aux milieux granulaires", Thèse de docteur de l'université de Lille I, 2000 